\title{
PERFIL EPIDEMIOLÓGICO DE GESTANTES DE ALTO RISCO COM PARTO PREMATURO EM UM HOSPITAL DE REFERÊNCIA
}

\author{
Jacqueline Vergutz Menetrier \\ Mestranda no Programa de Mestrado Profissional em \\ Plantas Medicinais e Fitoterápicos na Atenção Básica \\ na Universidade Paranaense (UNIPAR), Docente do \\ departamento de enfermagem da Universidade Para- \\ naense (UNIPAR), Paraná, Brasil.
}

E-mail: jacquevergutz.menetrier@hotmail.com

\section{Gleidaiane de Almeida}

Discente do curso de enfermagem da Universidade Paranaense (UNIPAR), Paraná, Brasil.

\begin{abstract}
RESUMO: O estudo objetivou identificar o perfil epidemiológico das gestantes classificadas como sendo de alto-risco internadas em um hospital de referência no Sudoeste do Paraná que tiveram parto prematuro durante o ano de 2015. Trata-se de uma pesquisa de campo de caráter retrospectivo e documental, com abordagem quantitativa, cujas informações foram coletadas dos prontuários das pacientes que constituíram a amostra. Durante o período estudado, foram registrados 820 partos na instituição, desses, 153 ocorreram prematuramente. Assim, a taxa de prematuridade na instituição, durante o período estudado foi de 18,6\%. Quanto aos dados sociodemográficos, foram encontrados em maior frequência idades entre 17 e 25 anos (41,5\%), com ensino médio completo e com companheiro. Em relação às características da assistência, prevaleceram as mulheres que realizaram mais de 6 consultas de pré-natal $(64,6 \%)$, tendo iniciado ainda no primeiro trimestre $(76,2 \%)$, sendo que o parto ocorreu entre 33 e 36 semanas de gestação em $81,5 \%$ dos casos e através de cesárea. Faz-se necessário adotar medidas que possibilitem a identificação precoce de possíveis fatores desfavoráveis para a evolução da gestação, tratando de forma adequada cada situação, viabilizando uma gestação sem maiores complicações para a saúde da mãe e do bebê.
\end{abstract}

PALAVRAS-CHAVE: Perfil de Saúde; Gravidez de Alto Risco; Trabalho de Parto.

\section{EPIDEMIOLOGICAL PROFILE OF HIGH-RISK PREGNANT FEMALES WITH PREMATURE BIRTH IN A REFERENCE HOSPITAL}

\begin{abstract}
The epidemiological profile of high-risk pregnant females with premature birth in 2015, hospitalized in a reference hospital in the south-western region of the state of Paraná, Brazil, is identified. Retrospective, quantitative and documental field research retrieved data from sampled patients' clinical charts. Analysis comprised 820 births in the institution of which 153 were premature. Prematurity rate in the institution during the period under analysis reached $18.6 \%$. Socio-demographic data revealed greater frequency within the $17-25$ years bracket (41.5\%), with complete high schooling and a partner. Females who had 6 prenatal visits were predominant (64.6\%), starting from the first trimester (76.2\%), with birth between the 33rd and 36th week of pregnancy $(81.5 \%)$ by caesarian incision. Early identification of possible unfavorable factors for pregnancy should be taken, with adequate care for each condition for a pregnancy without health complications for the mother and child.
\end{abstract}

KEY WORDS: Health profile; Pregnancy; High-risk; Labor; Obstetric. 


\section{INTRODUÇão}

Oprocessogestacional trata-se deuma experiência de vida saudável que engloba mudanças físicas, sociais e mentais no cotidiano das gestantes e também das pessoas que com elas convivem. Em algumas situações, a gestação pode trazer riscos para o processo saúde-doença da mãe e do bebê através de uma evolução desfavorável da gravidez, podendo estar relacionado a fatores maternos ou fetais, caracterizando a gestação como de alto-risco. Deve-se levar em consideração, que nem todos os indivíduos têm a mesma probabilidade para adoecer ou morrer, então, não são todas as gestações que oferecem riscos para a mãe ou para a criança (BRASIL,2012a).

Dentre os principais problemas decorrentes da gestação de alto-risco, destaca-se o parto prematuro ou pré-termo, que é o nascimento do bebê entre 22 e 37 semanas incompletas de gestação. Esse tipo de parto é responsável por mais de $75 \%$ da mortalidade e da morbidade entre os recém-nascidos. No Brasil, a média de prematuridade fica em torno de $12,3 \%$. Os maiores índices encontrados foram na região Nordeste, com $14,7 \%$ e os menores na região Sudeste, com $11,1 \%$ do total (LAJOS, 2014).

Para analisar os nascimentos prematuros utiliza-se a taxa de prematuridade que é definida como o número de nascidos vivos prematuros em relação ao total de nascidos (vivos e mortos) da operadora no ano considerado. Esse indicador tem como objetivo verificar as variações temporais do indicador, por operadora, identificando tendências e situações de desigualdade que possam demandar a realização de estudos especiais (BRASIL, 2012b).

A prematuridade é decorrente de circunstâncias diversas e imprevisíveis, em todos os lugares e classes sociais, afetando diretamente a estrutura familiar alterando as expectativas e anseios que permeiam a perinatalidade. Muitos podem ser os componentes que influenciam e são influenciados pelo complexo processo do nascimento prematuro. Por isso, conhecer as causas que levam ao parto prematuro tem tornado-se prioridade para as equipes de saúde no que diz respeito à morbimortalidade materno e infantil (RAMOS; CUMAN, 2009).

A morbimortalidade materna e perinatal ainda continua elevada no Brasil, incompatíveis com o atual nível de desenvolvimento econômico e social do país. Sabe-se que a maioria das mortes e complicações que surgem durante a gestação, parto e puerpério são preveníveis, mas, para isso é necessária a participação ativa do sistema de saúde. Assim, a mãe e sua família também devem receber aconselhamento sobre a morbidade e mortalidade associadas à idade gestacional e à eficácia limitada do tratamento, afim de prevenir os partos prematuros (BRASIL, 2012a).

Desta forma, o presente estudo teve como objetivo identificar o perfil epidemiológico das gestantes, bem como, as comorbidades presentes nas gestantes classificadas como sendo de alto-risco internadas em um hospital de referência no Sudoeste do Paraná, que tiveram parto prematuro durante o ano de 2015.

\section{METODOLOGIA}

Trata-se de uma pesquisa de campo de caráter retrospectivo e documental, com abordagem quantitativa, realizada no Hospital Regional do Sudoeste (HRS) Walter Alberto Pecóits, localizado na cidade de Francisco Beltrão no Sudoeste do Paraná. Os dados foram coletados diretamente dos prontuários das pacientes que estiveram internadas no Hospital Regional do Sudoeste e tiveram parto prematuro durante o período de janeiro de 2015 a dezembro de 2015. Os prontuários que não continham todas as variáveis estudadas foram excluídos.

O Hospital Regional do Sudoeste Walter Alberto Pecóits (HRS) foi inaugurado em 26 de fevereiro de 2010, sendo referência no atendimento à gestação de risco intermediário e alto. Atende pessoas referenciadas, preferencialmente das $7^{\mathrm{a}}$ e $8^{\mathrm{a}}$ regionais de saúde, localizadas nos municípios de Pato Branco e Francisco Beltrão, atendendo ao todo 42 municípios. Tem 100\% dos seus atendimentos através do Sistema Único de Saúde (SUS) (SESA, 2011).

A coleta de dados aconteceu mediante autorização da instituição através da Declaração de Permissão de Utilização de Dados e aprovação do Comitê de Ética em Pesquisa Envolvendo Seres Humanos (CEPEH) da Universidade Paranaense - Unipar através do 
parecer número 1.522.030. De acordo com a Resolução do Conselho Nacional de Saúde 466/2012.

A pesquisa de campo foi realizada após a autorização do responsável pelo Serviço de Armazenamento Médico e Estatístico do HRS, respeitando datas e horários estabelecidos pelo responsável do local. A presente pesquisa dispensou Termo de Consentimento Livre Esclarecido (TCLE), pois, trata-se de um estudo de caráter documental, cujos dados foram coletados apenas dos prontuários das pacientes selecionadas para o estudo, não havendo contato direto com elas.

Para a coleta dos dados, foi utilizado um roteiro previamente elaborado pela pesquisadora, que serviu como norteador das informações coletadas do prontuário das pacientes selecionadas para participar do estudo. O roteiro é composto de 20 perguntas, escolaridade, idade materna e situação conjugal; dados obstétricos da mãe (idade gestacional no momento do parto, $\mathrm{n}^{\circ} \mathrm{de}$ gestações anteriores, $\mathrm{n}^{0}$ de partos vaginais, $\mathrm{n}^{0}$ de partos por cesárea, $\mathrm{n}^{\mathrm{O}}$ de nascidos vivos, $\mathrm{n}^{\mathrm{O}}$ de nascidos mortos $\mathrm{e}$ $\mathrm{n}^{\mathrm{o}}$ de perdas fetais-abortos); características da assistência ( $\mathrm{n}^{0}$ de consultas do pré-natal, mês da gestação que começou o pré-natal, intercorrências gestacionais-quais, tipo de parto); e características do recém-nascido (peso ao nascer e índice de Apgar no $1^{\circ}$ minuto e $5^{\circ}$ minuto).

Para calcular a taxa de prematuridade, foi utilizada a fórmula indicada pelo Ministério da Saúde, na qual o número de nascidos prematuros (153) foi dividido pelo número total de nascidos no ano de 2015 (820) e multiplicado o valor por 100 (BRASIL, 2012b).

Os dados coletados foram submetidos à análise descritiva utilizando o programa Statistical Package for Social Science (SPSS®) versão 21.0 (Windows; Chicago, EUA).

\section{RESULTADOS}

Durante o período estudado, de janeiro a dezembro de 2015 , foram registrados 820 partos na instituição, desses, 153 ocorreram prematuramente, ou seja, com menos de 37 semanas de gestação. Assim, a taxa de prematuridade na instituição, durante o período estudado foi de $18,6 \%$. Dos 153 prontuários de mulheres com parto prematuro, foram analisados 130 , já que 23 foram excluídos por não conter todas as informações necessárias à pesquisa.

Com o objetivo de conhecer o perfil da amostra, foram coletados dados sociodemográficos das gestantes que tiveram parto prematuro, apresentados na tabela 1. Conforme pode ser observado na tabela, o maior número de partos prematuros ocorreu em mulheres que possuíam entre 17 e 25 anos (41,5\%), seguidos das mulheres com 26 a 35 anos (36,2\%) com ensino médio completo $(37,7 \%)$ e com companheiro $(86,9 \%)$.

Tabela 1. Perfil sociodemográfico de gestantes de alto risco com parto prematuro em um hospital de referência no ano de 2015

\begin{tabular}{lcc}
\hline \multicolumn{1}{c}{ VARIÁVEL $(\mathbf{n = 1 3 0 )}$} & $\mathbf{n}^{\mathbf{0}}$ & $\%$ \\
\hline Idade & 9 & 6,9 \\
\hline Menor que 17 & 54 & 41,5 \\
17 a 25 anos & 47 & 36,2 \\
26 a 35 anos & 20 & 15,4 \\
36 anos ou mais & & \\
\hline Escolaridade & 42 & 32,4 \\
\hline Fundamental completo & 12 & 9,2 \\
Fundamental incompleto & 49 & 37,7 \\
Médio completo & 3 & 2,3 \\
Médio incompleto & 15 & 11,5 \\
Superior completo & 9 & 6,9 \\
Superior incompleto & & \\
\hline Situação Conjugal & 113 & 86,9 \\
\hline Com companheiro & 17 & 13,1 \\
\hline Sem companheiro & & \\
\hline Fonte: Dados da pesquisa. & &
\end{tabular}

Fonte: Dados da pesquisa.

Em relação aos dados do histórico obstétrico, observe a tabela 2. Diante dos dados apresentados, é possível observar que houve maior frequência de partos prematuros em mulheres primigestas $(46,9 \%)$, juntamente com mulheres entre 1 e 3 gestações anteriores $(46,9 \%)$, que não haviam passado por parto vaginal $(67,7 \%)$ e nem parto cesáreo $(77,7 \%)$, que não possuíam nenhum nascido vivo ou morto $(50 \%$ e $98,5 \%$, 
respectivamente) e sem histórico de aborto (82,3\%).

Tabela 2. Histórico obstétrico de gestantes de alto risco com parto prematuro em um hospital de referência no ano de 2015

\begin{tabular}{|c|c|c|}
\hline VARIÁVEL $(n=130)$ & $\mathbf{n}^{\mathbf{o}}$ & $\%$ \\
\hline \multicolumn{3}{|l|}{$\mathbf{N}^{0}$ gestações anteriores } \\
\hline Nenhuma & 61 & 46,9 \\
\hline 1 a 3 gestações & 61 & 46,9 \\
\hline 4 a 5 gestações & 7 & 5,4 \\
\hline Mais de 5 gestações & 1 & 0,8 \\
\hline \multicolumn{3}{|l|}{$\mathrm{N}^{0}$ partos vaginais } \\
\hline Nenhum & 88 & 67,7 \\
\hline $1 \mathrm{a} 2$ partos & 37 & 28,5 \\
\hline 3 a 4 partos & 3 & 2,3 \\
\hline Mais de 4 partos & 2 & 1,5 \\
\hline \multicolumn{3}{|l|}{$\mathrm{N}^{0}$ parto cesáreo } \\
\hline Nenhum & 101 & 77,7 \\
\hline 1 a 2 cesáreas & 29 & 22,3 \\
\hline \multicolumn{3}{|l|}{$N^{0}$ nascidos vivos } \\
\hline Nenhum & 65 & 50,0 \\
\hline $1 \mathrm{a} 2$ & 60 & 46,2 \\
\hline $3 \mathrm{a} 4$ & 3 & 2,3 \\
\hline Mais que 4 & 2 & 1,5 \\
\hline \multicolumn{3}{|l|}{$\mathrm{N}^{\mathrm{o}}$ de nascidos mortos } \\
\hline Nenhum & 128 & 98,5 \\
\hline $1 \mathrm{a} 2$ & 2 & 1,5 \\
\hline \multicolumn{3}{|l|}{$\mathrm{N}^{\mathrm{o}}$ de abortos } \\
\hline Nenhum & 107 & 82,3 \\
\hline $1 \mathrm{a} 2$ & 20 & 15,4 \\
\hline $3 \mathrm{a} 4$ & 3 & 2,3 \\
\hline
\end{tabular}

Fonte: Dados da pesquisa.

A tabela 3 apresenta os dados referentes às características da assistência prestada as gestantes. Diante dos dados apresentados, observa-se que relacionado à característica da assistência, os partos prematuros ocorreram com maior frequência em mulheres com idade gestacional de 33-36 semanas (81,5\%), que realizam mais de 6 consultas de pré-natal (64,6\%), com início das consultas ainda no $1^{\circ}$ trimestre da gestação $(76,2 \%)$.
Tabela 3. Características da assistência prestada a gestantes de alto risco com parto prematuro em um hospital de referência no ano de 2015

\begin{tabular}{|c|c|c|}
\hline VARIÁVEL (n=130) & $\mathbf{n}^{\mathbf{o}}$ & $\%$ \\
\hline \multicolumn{3}{|l|}{ Duração da gestação } \\
\hline 25 a 29 semanas & 10 & 7,7 \\
\hline 30 a 32 semanas & 14 & 10,8 \\
\hline 33 a 36 semanas & 106 & 81,5 \\
\hline \multicolumn{3}{|l|}{$\mathrm{N}^{0}$ consultas pré-natal } \\
\hline 0 a 3 consultas & 10 & 7,7 \\
\hline 4 a 6 consultas & 36 & 27,7 \\
\hline Mais que 6 consultas & 84 & 64,6 \\
\hline \multicolumn{3}{|l|}{ Início do pré-natal } \\
\hline $1^{\circ}$ trimestre & 99 & 76,2 \\
\hline $2^{\circ}$ trimestre & 17 & 13,1 \\
\hline $3^{\circ}$ trimestre & 4 & 3,1 \\
\hline Não fez & 2 & 1,5 \\
\hline Ignorado & 8 & 6,1 \\
\hline \multicolumn{3}{|l|}{ Intercorrências gestacionais } \\
\hline Nenhuma & 1 & 0,8 \\
\hline TPP (trabalho de parto prematuro) & 26 & 20,0 \\
\hline Alterações do bem-estar fetal & 17 & 13,1 \\
\hline ITU (infecção do trato urinário) & 9 & 6,9 \\
\hline Síndromes hipertensivas & 13 & 10,0 \\
\hline Alterações volume do líquido amniótico & 26 & 20,0 \\
\hline Desvio do crescimento fetal & 13 & 10,0 \\
\hline Síndromes hemorrágicas & 6 & 4,6 \\
\hline Outras & 19 & 14,6 \\
\hline \multicolumn{3}{|l|}{ Tipo de parto } \\
\hline Parto vaginal & 30 & 23,1 \\
\hline Parto cesáreo & 100 & 76,9 \\
\hline
\end{tabular}

Fonte: Dados da pesquisa

Sobre as intercorrências neste período, destacouse o trabalho de parto prematuro (TPP), juntamente com as alterações no volume do líquido amniótico (ambos com 20\%). Outras intercorrências observadas foram diabetes mellitus gestacional (DMG), hipotireoidismo, hipertireoidismo, obesidade mórbida, depressão e esquizofrenia. 
Em relação ao tipo de parto, o parto cesáreo ocorreu em $76,9 \%$ dos casos.

A tabela 4 apresenta as características dos recémnascidos. É possível observar que grande parte dos prematuros nasceu com peso entre 1500 e 2500 gramas (56,9\%), obtendo índice de Apgar no $1^{\circ}$ e $5^{\circ}$ minuto de vida de 7 e 10 ( $81,5 \%$ e $96,8 \%$ respectivamente).

Tabela 4. Características do recém-nascido gestantes de alto risco com parto prematuro em um hospital de referência no ano de 2015

\begin{tabular}{lcc}
\hline \multicolumn{1}{c}{ VARIÁVEL $(\mathbf{n = 1 3 0 )}$} & $\mathbf{n}^{\mathbf{0}}$ & $\%$ \\
\hline Peso do RN & & \\
\hline Menos de 1500 gramas & 33 & 25,4 \\
1500 a 2500 gramas & 74 & 56,9 \\
Acima de 2500 gramas & 23 & 17,7 \\
\hline Apgar no $\mathbf{1}^{\mathbf{0}}$ minuto de vida & & \\
\hline Menor que 7 & 24 & 18,5 \\
De 7 a 10 & 106 & 81,5 \\
\hline Apgar no $\mathbf{5}^{\mathbf{0}}$ minuto de vida & & \\
\hline Menos de 7 & 5 & 3,2 \\
De 7 a 10 & 125 & 96,8 \\
\hline
\end{tabular}

Fonte: dados da pesquisa.

\section{DISCUSSÃO}

No presente estudo, a taxa de prematuridade encontrada, referente ao ano de 2015, foi de 18,6\%. Já em estudo realizado na Universidade Estadual de Campinas (UNICAMP) sobre a prematuridade no Brasil, encontrouse uma taxa de prematuridade nacional de 12,3\% (LAJOS, 2014). Nas últimas décadas tem se observado aumento gradual dos nascimentos pré-termo, tanto nacionalmente quanto internacionalmente. Os fatores associados a esse aumento são diversos, porém, destaca-se o aumento da intervenção médica nos nascimentos como, por exemplo, o crescente número de cesarianas. Apesar do aumento progressivo de partos prematuros, também evidenciase aumento na taxa de prematuros sobreviventes e da sobrevida desses, a literatura destaca que isso ocorre, principalmente, pelos avanças tecnológicos e esforços colaborativos da equipe de saúde no atendimento do binômio mãe e recém-nascido (BETTIOL; BARBIERI; DA SILVA, 2010).

Acredita-se que um dos fatores que podem estar relacionados a elevada taxa de prematuridade encontrada no estudo, é que o Hospital Regional da Sudoeste é referência em gestação de alto risco, para os 42 municípios das $7^{\mathrm{a}}$ e $8^{\mathrm{a}}$ regionais de saúde do Paraná, assim, espera-se um número maior de partos prematuros.

A gestação de alto-risco é definida como aquela onde existe um ou mais fatores de risco para a vida/saúde da gestante ou do bebê, que pode afetar adversamente seu resultado. Compreende uma série bastante ampla de condições clínico-obstétricas que poderão trazer complicações à gravidez, consideradas situações de risco real, ou ainda, condições pré-existentes, capazes de em algum momento tornar-se danosas para a evolução saudável da gestação constituindo um risco potencial. Dentre os principais problemas decorrentes da gestação de alto-risco, destaca-se o parto prematuro ou prétermo, pois ele é a condição mais desfavorável para a sobrevida do bebê fora do útero, no entanto, em algumas circunstâncias, ele é um desfecho inevitável (CASCAES et al., 2008)

Quando se trata de parto prematuro, várias podem ser as causas para seu desencadeamento, inclusive as condições socioeconômicas da gestante. Essas podem afetar de várias formas o desenvolvimento da gestação, já que muitas vezes, as mulheres são jovens não possuem uma escolaridade elevada, e em alguns casos não contam com o apoio de um cônjuge. Desta forma, ficam desprotegidas e carentes de informações. Em alguns casos, essas mulheres acabam não sabendo ao certo quando sua gravidez está correndo dentro da normalidade e quando necessário podem não saber onde buscar apoio, bem como, quais são os cuidados básicos a serem seguidos para que a gestação se desenvolva sem grandes problemas (HACKBARTH et al., 2015).

Neste estudo, relacionado aos dados sociodemográficos da gestante, os resultados encontrados foram maior frequência de mulheres com idades entre 17 e 25 anos, com ensino médio completo e com companheiro. Esses resultados corroboram com os encontrados por outro estudo desenvolvido na Fundação Santa Casa de Misericórdia, no Pará, em 2010, no qual 
a casuística foi composta em sua maioria por puérperas na faixa etária entre 19 e 24 anos (36,0\%) e com ensino médio completo (41,0\%) (SOUZA; BOTELHO; MACEDO, 2011).

Referente às características da assistência, os dados encontrados nesta pesquisa foram de duração da gestação entre 33 e 36 semanas. Tais resultados assemelham-se aos encontrados por outro estudo realizado em um município do sul de Minas Gerais de 2005 a 2009, em que verificou-se que $85,4 \%$ dos partos ocorreram entre a $32^{\circ}$ e a $36^{\circ}$ semana de gestação. Desta forma, é possível observar que embora os partos tenham ocorrido prematuramente, estavam próximos do término (SILVA et al., 2013).

No que se relaciona à idade gestacional, o desenvolvimento fetal é dividido em fases, nos primeiros dois trimestres da gestação todos os órgãos do bebê são formados. No $3^{\circ}$ trimestre, da $29^{\circ}$ semana até o nascimento, o bebê principalmente ganha peso, aproximadamente 300 gramas por semana. Desta forma, o nascimento prematuro não permite em grande parte dos casos que o bebê nasça com peso adequado, sendo caracterizado com baixo-peso (FEBRASGO, 2011).

$O$ fato de os partos terem ocorrido em sua maior parte próximos do término pode ser explicado pelo uso de métodos que proporcionam o prolongamento da gestação, até que o bebê encontre-se em condições de viver fora do útero, como por exemplo a tocólise, em que, durante o TPP, são inibidas as contrações uterinas através da utilização de medicamentos, adiando o nascimento. Quando isso ocorre, é possível fazer o uso de corticóide para amadurecer o pulmão do bebê, desta forma, quando nascer, apresentará melhores condições de sobrevida extrauterina (BITTAR; ZUGAIB, 2010).

No presente estudo, acredita-se que o nascimento próximo do término da gestação é explicado pela existência e uso desses métodos necessários para adiar o nascimento dos bebês, pois, trata-se de uma referência para gestação de alto risco, a partir das equipes de multiprofissionais que são capacitados para atuar em tais situações. Caso o estudo tivesse sido realizado em outro ambiente, os dados encontrados poderiam ser diferentes.

Observou-se que 64,6\% das gestantes passaram por mais de 6 consultas de pré-natal, que é o número mínimo de consultas preconizadas pelo Ministério da Saúde, tendo dado seu início ainda no primeiro trimestre de gestação. Tais dados corroboram com os resultados encontrados em um estudo desenvolvido em uma maternidade de referência ao atendimento e parto de alto risco da rede SUS, em Teresina no Piauí, no período de outubro de 2012 e abril de 2013. Os dados encontrados por eles indicaram consultas de pré-natal feitas de maneira adequada, com média de 7 consultas de cada gestante, que se iniciaram ainda no primeiro trimestre da gravidez (GONZAGA et al., 2016).

Para saber se a gestação é de baixo, médio ou alto-risco, é necessário que a gestante realize as consultas de pré-natal, que tem por objetivo assegurar o desenvolvimento favorável da gestação, permitindo o parto de um recém-nascido saudável, sem impacto para a saúde materna, abordando aspectos psicossociais, atividades educativas e preventivas. Recomenda-se que toda gestante realize no mínimo 6 consultas de acompanhamento pré-natal. Deve ser iniciado ainda no primeiro trimestre afim de identificar precocemente possíveis fatores desfavoráveis ou de risco, possibilitando que a gestação ocorra da forma mais saudável possível (BRASIL, 2010).

Quanto às intercorrências gestacionais, destacaram-se as alterações no volume de líquido amniótico, bem como, no Trabalho de Parto prematuro.

As alterações do volume do líquido amniótico ocorrem quando o líquido aumenta demasiadamente (polidrâmnio), ou encontra-se em quantidade abaixo do valor normal (oligodrâmnio). Seu volume normal varia de acordo com o tempo de gestação, reduzindose fisiologicamente nas últimas semanas. Tais alterações são fatores de risco para o bem-estar fetal, pois, o líquido é responsável pelo desenvolvimento saudável do feto dentro do útero, prevenindo complicações como traumas mecânicos e ajudando no desenvolvimento musculoesquelético do feto, e no amadurecimento pulmonar (PINHEIRO et al., 2014).

O parto prematuro é a principal causa de morbidade e mortalidade neonatal. Cerca de $75 \%$ dos nascimentos prematuros são decorrentes do trabalho de parto espontâneo. O diagnóstico correto do TPP nem sempre é fácil e classicamente baseia-se na presença de contrações uterinas regulares (pelo menos uma a cada 
5 minutos) e persistentes, dilatação cervical igual ou superior a $1 \mathrm{~cm}$, esvaecimento cervical igual ou superior a $80 \%$ e progressão das alterações cervicais. Em um falso trabalho de parto verificar-se-ia apenas o aparecimento de contrações irregulares e sem coordenação, além da ausência de modificações importantes no colo uterino (BITTAR; ZUGAIB, 2010).

Sobre o tipo de parto, a maior parte dos nascimentos prematuros ocorreu através de cesárea (76,9\%). Tais resultados corroboram com os fornecidos pelo Ministério da Saúde, sendo que o Brasil ocupa a $1^{\circ}$ colocação na realização de cesáreas no mundo todo, representando 57\% do total dos partos (LAJOS, 2014).

Embora em sua grande maioria, os autores marginalizem a escolha do parto cesáreo, existem condições específicas em que ele é necessário, como em casos nos quais os bebês são prematuros extremos. Por possuírem peso muito baixo, as contrações do parto natural acabam por lhe causar sofrimento fetal e hipóxia, algumas vezes, quando ele está pélvico, a pressão da cabeça do bebê contra a pelve materna acaba causando hemorragia intracraniana no bebê (BITTAR; ZUGAIB, 2005)

De acordo com o Manual Técnico de Gestação de Alto Risco, a gravidez de risco não é sinônimo de cesariana, sendo possível a indução ao parto vaginal ou mesmo aguardar seu início espontaneamente. Porém, sabe-se que não é isso o que ocorre atualmente no Brasil, na maioria dos casos, cesáreas eletivas são programadas apenas por serem mais cômodas para os médicos, o que acaba elevando os índices de cesarianas, ficando acima do que recomenda a Organização Mundial da Saúde. Além disso, atualmente, o parto cesáreo está associado com a maior frequência de infecção e de hemorragias maternas, e de síndrome do desconforto respiratório do recémnascido (BRASIL, 2012a).

De acordo com a portaria 466 de 14 de julho de 2000, no Brasil, o limite de cesarianas é estabelecido por estado, sendo que no Paraná, onde o presente estudo foi realizado, esse limite é de $35 \%$ do total dos partos (BRASIL, 2000).

Depois disso, segundo o Ministério da Saúde, são classificados como baixo peso ao nascer, bebês com menos de 2500 gramas, sejam eles prematuros ou não.

Em relação às características do recém-nascido, no presente estudo os pesos foram entre $1500 \mathrm{~g}$ a 2500g, e com Apgar de 7 a 10 no $1^{\circ}$ e $5^{\circ}$ minutos de vida respectivamente. Dados estes semelhantes aos encontrados em pesquisa desenvolvida em Guarapuava no ano de 2005 e publicados em 2009, que demonstraram RNs pesando entre $2000 \mathrm{~g}$ e $2500 \mathrm{~g}$, obtendo Apgar entre 7 e 10 no $1^{\circ}$ e $5^{\circ}$ minutos de vida (RAMOS; CUMAN, 2009).

Para avaliar as condições do nascimento do RN, além do peso ao nascer, é avaliado o Índice de Apgar, que é comumente empregado para avaliar o ajuste imediato do recém-nascido à vida extrauterina, avaliando suas condições de vitalidade. Consiste na avaliação de 5 itens do exame físico do recém-nascido, com 1, 5 e 10 minutos de vida. Os aspectos avaliados são: frequência cardíaca, esforço respiratório, tônus muscular, irritabilidade reflexa e cor da pele. Para cada um dos 5 itens é atribuída uma nota de 0 a 2 . Somam-se as notas de cada item e tem-se o total, que pode dar uma nota mínima de 0 e máxima de 10 (BRASIL, 2011).

Estes dados mostram que apesar de nascer prematuramente, os bebês em sua maioria têm boa vitalidade fora do útero, porém, é necessário salientar que embora tenham nascido em boas condições, podem apresentar problemas tardiamente, levando em consideração que a prematuridade é um fator relativamente responsável por grande parte das mortes neonatais e perinatais. Assim sendo, é extremamente importante que as gestantes tenham um acompanhamento efetivo da equipe multidisciplinar antes, durante e após a gestação, afim de prevenir à morbimortalidade materna, perinatal e infantil decorrente da assistência inadequada.

\section{CONCLUSÃO}

Os dados encontrados no estudo, como a elevada taxa de prematuridade e as características maternas e assistências relacionadas ao parto prematuro, devem ser considerados na definição de estratégias, principalmente no Sudoeste do Paraná para ações de controle que visem à diminuição da taxa de prematuridade, tendo em vista que o nascimento prematuro é um fator de risco para a morbimortalidade neonatal e também materna. Portanto, as equipes multidisciplinares devem trabalhar de forma 
coordenada e integrada, prestando assistência de maneira mais adequada possível para cada situação.

Assim, acredita-se que a realização de novos estudos na instituição envolvendo um maior período de tempo seria de grande valia, com a finalidade de traçar um perfil mais exato da prematuridade local, tendo em vista que algumas informações relevantes para que o objetivo da pesquisa fosse alcançado de maneira satisfatória não foram encontradas.

\section{REFERÊNCIAS}

BETTIOL, H.; BARBIERI, M.A.; DA SILVA, A.A.M. Epidemiologia do nascimento pré-termo: tendências atuais. Rev Bras Ginecol Obstet., v. 32, n. 2, p.57-60. 2010.

BITTAR. R. E; ZUGAIB. M. Condutas para o trabalho de parto prematuro. Rev Bras Ginecol Obstet., v. 27, n. 9, p. 561-566, 2005.

BITTAR. R. E.; ZUGAIB. M. Qual é a melhor via de parto para feto prematuro? Rev Femina, v. 38, p. 543-546, Brasília, 2010.

BRASIL. Ministério da Saúde. Portaria n. 466 de 14 de junho de 2000. Brasília, 2000.

BRASIL. Ministério da Saúde. Cadernos de Atenção Básica: Atenção ao pré-natal de alto risco. Brasília, 2010.

BRASIL. Ministério da Saúde. Atenção à saúde do recémnascido: guia para os profissionais de saúde. Brasília- DF, 2011. v. 1.

BRASIL. Ministério da Saúde. Cadernos de Atenção Básica: Atenção ao pré-natal de baixo risco. Brasília, 2012a.

BRASIL. Ministério da Saúde. Saúde Brasil 2011: Uma análise da situação de saúde e a vigilância da saúde da mulher. Brasília, 2012b.

CASCAES, A. M. et al. Prematuridade e fatores associados no Estado de Santa Catarina, Brasil, no ano de 2005: análise de dados do Sistema de Informações Sobre Nascidos Vivos. Cad. Saúde Pública, Rio de Janeiro, p. 1024-1032, maio 2008.

FEBRASGO. Federação Brasileira das Associações de
Ginecologia e Obstetrícia. Manual de orientação de gestação de alto risco. Brasília, 2011.

GONZAGA, I.C.A.; SANTOS, S.L.D.; SILVA, A.R.V.; CAMPELO, V. Atenção pré-natal e fatores de risco associados à prematuridade e baixo peso ao nascer em capital do nordeste brasileiro. Ciênc. Saúde Colet., Rio de Janeiro, v. 21, n. 6, jun. 2016.

HACKBARTH, B. B.; FERREIRA, J.A.; CARSTENS, H. P.; AMARAL, A.R.; SILVA, M.R.; SILVA, J.C.; FRANÇA, P.H.C. Suscetibilidade à prematuridade: investigação de fatores comportamentais, genéticos, médicos e sociodemográficos. Rev Bras Ginecol Obstet., v. 37, n. 8, p. 353-358, 2015.

LAJOS, G.J. Estudo multicêntrico de investigação em prematuridade no Brasil: implementação, correlação interclasse e fatores associados à prematuridade espontânea. Campinas SP: [s.n.], 2014.

PINHEIRO, S. N. et al. Oligodrâmnio e polidramnio: atuação do enfermeiro. In: CONGRESSO BRASILEIRO DE ENFERMAGEM, 2014, Pará. Anais.... out. 2014.

RAMOS, H. A. C.; CUMAN, R. K. N. Fatores de Risco para Prematuridade: Pesquisa documental. Esc. Anna Nery., v. 13, n. 2, p. 297-304, abr.jun. 2009.

SESA. Hospital Regional do Sudoeste Walter Alberto Pecóits. Relatório de atividades 2011. Francisco Beltrão: SESA, 2011.

SILVA, R. P. et al. Prematuridade: características maternas e neonatais segundo dados do sistema de informações sobre nascidos vivos. Rev Enferm UFPE online, Recife, v. 7, n. 5, p. 1349-1355, maio 2013.

SOUZA, L. F.; BOTELHO, N. M.; MACEDO, N. Fatores de risco para o parto prematuro em puérperas que tiveram parto pretermo. Rev. Para. Med., v. 25. n. 4. out./dez. 2011.

Recebido em:13 de outubro de 2016

Aceito em: 27 de fevereiro de 2017 
